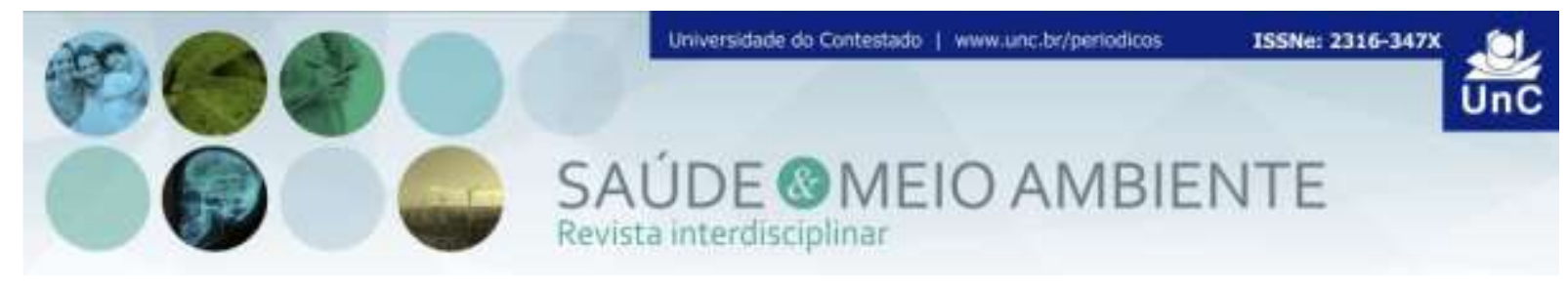

\title{
DESENVOLVIMENTO BIOPSICOSSOCIAL DE ADULTOS COM MIELOMENINGOCELE
}

\section{BIOPSYCHOSOCIAL DEVELOPMENT OF ADULTS WITH MYELOMENINGOCELE}

\author{
Beatriz Essenfelder Borges ${ }^{1}$ \\ Mayara da Silva Kovalhuk ${ }^{2}$
}

\begin{abstract}
RESUMO
A Mielomeningocele é uma doença decorrente da malformação do tubo neural nas primeiras semanas de gestação. Não possui cura, porém são proporcionados tratamentos ao longo do desenvolvimento para evitar complicações irreversíveis. Objetivo: Mostrar a atual qualidade de vida dos portadores de mielomeningocele e os obstáculos ao longo do seu desenvolvimento. Método: Trata-se de uma pesquisa descritiva exploratória de natureza qualitativa, com cinco indivíduos com sequelas da Mielomeningocele maiores de 18 anos, que foram entrevistados. Resultado: A análise dos dados coletados foi realizada com o apoio da literatura pré-existente, os resultados permitiram compreender o impacto diário desta malformação no desenvolvimento e as dificuldades enfrentadas ao longo dos anos. Conclusão: A partir dos relatos coletados foi possível compreender as limitações desenvolvidas diante das sequelas secundárias, assim como a superação e adaptação dos indivíduos com mielomeningocele ao longo do seu desenvolvimento.
\end{abstract}

Palavras-chave: Mielomeningocele. Qualidade de vida. Perfil de impacto.

\begin{abstract}
Myelomeningocele is an intrauterine disease resulting from malformation of the neural tube in the first weeks of pregnancy. There is no cure, but treatments are provided throughout development to avoid irreversible complications. Objective: To show the current quality of life of patients with myelomeningocele and the obstacles along their development. Method: This is a descriptive exploratory research of qualitative nature, with five patients with myelomeningocele over 18 years, through questions open to discussion. Results: The analysis of the collected data was performed with the support of pre-existing literature. The results allowed us to understand the daily impact of this malformation on development and the difficulties overcome over the years. Conclusion: From the collected reports it was possible to understand the limitations

\footnotetext{
${ }^{1}$ Doutorado em Microbiologia, Parasitologia e Patologia pela Universidade Federal do Paraná, Brasil. Professora do Faculdades Pequeno Príncipe. Paraná. Brasil. E-mail: biaessenfelder@gmail.com pela Faculdade Integradas Santa Cruz (FARESC). Paraná. Brasil. E-mail: mayarakovalhuk15@hotmail.com.
} ${ }^{2}$ Especialização em andamento em Estomaterapia, Faculdade UNIBF, Graduação em Enfermagem,
\end{abstract}


developed in the face of secondary diseases, as well as the overcoming and adaptation of myelomeningocele patients throughout their development.

Keywords: Myelomeningocele. Quality of life. Impact profile.

\section{INTRODUÇÃO}

A Mielomeningocele (MMC) é uma malformação congênita no sistema nervoso central, com incidência entre as quatro primeiras semanas de gestação. Pode advir durante o fechamento do tubo neural ocasionando em doenças como Anencefalia e Encefalocele ou na região lombo-sacra gerando a Mielomeningocele, também conhecida como espinha bífida aberta, não oferecendo barreira de proteção contra o meio externo e expondo a medula espinal. ${ }^{1}$

De acordo com Baldisserotto e colaboradores (2010) "estima-se que no Brasil a incidência seja de aproximadamente 1,1: 1000 nascidos vivos". Sendo a causa de caráter indefinido e podendo estar relacionado a fatores tais como ambientais ou genéticos, supressão de vitaminas A, D e Ácido Fólico, atualmente considerado o maior fator de riscos para desencadeamento desta doença. ${ }^{2-3}$

Segundo Affonso e colaboradores (2014) "ácido fólico ajuda a formar as células brancas e vermelhas do sangue, além disso, ajuda na formação do material genético de cada célula". A falta desta vitamina durante a gestação podendo acarretar deformidades do tubo neural, ocasionadas na fase inicial do desenvolvimento fetal. Sendo recomendada uma suplementação antes e durante a gestação. ${ }^{4-5}$

Além disso, visando a disponibilidade limitada de informações sobre o desenvolvimento e qualidade de vida de indivíduos com mielomeningocele na vida adulta, esta pesquisa teve como intuito obter diferentes visões acerca do desenvolvimento, complicações secundárias e o bem-estar dos portadores desta deficiência atualmente.

Tendo em vista as sequelas ocasionadas por esta deficiência, buscamos a individualidade de percepção de cada voluntário, baseado em experiências particulares vividas ao longo dos anos. Posteriormente os dados coletados foram interpretados, analisados junto à literatura já existente e categorizados para melhor compreensão.

Dessa maneira, o objetivo desse trabalho foi identificar a qualidade de vida de adultos com mielomeningocele, já que a maioria dos artigos publicados retratam apenas crianças com sequelas dessa patologia. 


\section{METODOLOGIA}

Trata-se de uma pesquisa descritiva exploratória de natureza qualitativa, com intuito de observar diferentes parâmetros de desenvolvimento e aspectos distintos de uma situação, a partir de relatos de caso de adultos com mielomeningocele.

O estudo foi realizado na cidade de Curitiba-PR, com cinco indivíduos com mielomeningocele, com idade superior a dezoito anos. A escolha de voluntários nesta faixa etária se justifica pelo fato de que à medida que ocorre a transição da adolescência a maturidade, já que o indivíduo passa a estar menos exposto a procedimentos invasivos. Por exemplo, entre os 18 a 21 anos já atingiram a maturidade esquelética, não precisando de novas correções em joelho/pé por conta do crescimento. ${ }^{6}$

As entrevistas foram realizadas individualmente, conforme a disponibilidade de cada participante e assinatura prévia do Termo de Consentimento Livre e Esclarecido, realizando as devidas orientações e esclarecimentos a respeito da pesquisa.

Os dados foram posteriormente transcritos, tendo como base de análise a literatura já existente, sendo categorizada em seis tópicos para melhor compreensão. Não houve respostas assertivas ou incorretas, apenas a opinião pessoal de cada voluntário. Como garantia de anonimato dos voluntários entrevistados, foram identificados como voluntario (a), seguido de algarismo em ordem crescente (voluntario 1, voluntaria 2...).

A pesquisa foi aprovada pelo Comitê de Ética em Pesquisa em Seres Humanos do Hospital Paranaense de Otorrinolaringologia LTDA - IPO, sob- parecer $n^{\circ}$. 3.583.139.

\section{RESULTADOS E DISCUSSÃO}

Após as entrevistas foi realizada a categorização das respostas para melhor compressão dos dados obtidos, dessa maneira as categorias foram classificadas em números de 1 a 6 . Sendo a $1^{\circ}$ : Descrição socioeconômica, a $2^{\circ}$ Intervenções cirúrgicas, $3^{\circ}$ Apoio familiar no desenvolvimento, $4^{\circ}$ Principais obstáculos, $5^{\circ}$ Desenvolvimento profissional e o $6^{\circ}$ Relacionamentos afetivos.

\section{Categoria 1. Descrição social e de saúde dos voluntários}

As entrevistas foram realizadas com 5 voluntários adultos com mielomeningocele, com prevalência do sexo masculino. A idade dos voluntários variou entre 20 a 35 anos. A Tabela 1 resume as características das amostras coletadas. 
Tabela 1 - Descrição social.

\begin{tabular}{lccc}
\hline \multicolumn{1}{c}{ CATEGORIA } & VARIÁVEL & NÚMERO $^{\circ}$ & $\%$ \\
\hline SEXO & FEMININO & 1 & $80 \%$ \\
& MASCULINO & 4 & $20 \%$ \\
TOTAL: & 5 & $100 \%$ \\
& $20 \mathrm{~A} 25$ & 2 & $40 \%$ \\
& $26 \mathrm{~A} 30$ & 1 & $20 \%$ \\
ESCOLARIDADE & 31 A 35 & 2 & $40 \%$ \\
& TOTAL: & 5 & $100 \%$ \\
& ENSINO MÉDIO COMPLETO & 5 & $100 \%$ \\
& ENSINO SUPERIOR & 4 & $80 \%$ \\
& TOTAL: & 5 & $100 \%$
\end{tabular}

Fonte: Autor 2020

A Tabela 2 representa as condições clínicas e as sequelas associadas a mielomeningocele.

Tabela 2 - Condições clínicas e as sequelas associadas a mielomeningocele

\begin{tabular}{cccc}
\multicolumn{1}{c}{ CATEGORIA } & VARIÁVEL & NÚMERO $^{\circ}$ & $\%$ \\
\hline HIDROCEFALIA & SIM & 5 & $100 \%$ \\
& NÃO & 0 & $0 \%$ \\
CORREÇÃO COM & TOTAL: & 5 & $100 \%$ \\
DERIVAÇÕES & SIM & 5 & $100 \%$ \\
VENTRICULARES & NÃO & 0 & $0 \%$ \\
& TOTAL: & 5 & $100 \%$
\end{tabular}

Fonte: Dados subdivididos em categorias, a partir dos relatos coletados nas entrevistas, 2020.

Todos apresentaram sequela da mielomeningocele associada a incidência da hidrocefalia na infância. Castro e colaboradores (2010) relatam que em cerca de $90 \%$ dos casos de mielomeningocele podem ocorrer à hidrocefalia. ${ }^{7}$

A hidrocefalia ocorre quando o corpo produz excesso de líquido cefalorraquidiano (LCR) conhecido também como Liquor, fazendo com que este liquido não seja reabsorvido, ocasionando em uma pressão intracraniana.

Segundo Salomão e colaboradores (1995) "A maioria absoluta dos indivíduos com mielomeningocele têm hidrocefalia associada e, em mais de $70 \%$ dos casos, há necessidade de instalação de derivações ventriculares". ${ }^{8}$ 
Tendo isso em vista, todos os voluntários realizaram correção com a implantação cirúrgica de uma Derivação Ventrículo Peritoneal, mais conhecida com DVP, utilizado para aliviar a pressão intracraniana ocasionada pela Hidrocefalia atráves da drenagem deste líquido cefalorraquidiano para outra cavidade corporal. ${ }^{9}$

Quatro voluntários apresentaram sensibilidade a Látex, relatado ainda na infância. Segundo Yeh e colaboradores (2012) "crianças com mielomeningocele são as que mais se sensibilizam ao látex pelo contato muito frequente e precoce com cateteres, luvas, dentre outros". ${ }^{10}$

Atualmente todos os voluntários seguem em acompanhamento médico, porém com intervalo maior entre as avaliações, levando em conta as consultas contínuas de quando criança.

\title{
Categoria 2. Intervenções cirúrgicas
}

Os indivíduos com de mielomenigocele são submetidos a diversas intervenções cirúrgicas durante o seu desenvolvimento, sejam elas intrauterinas ou até mesmo nas primeiras horas após o seu nascimento, assim que avaliado e estabelecido às condições clínicas gerais do recém-nato. Tendo como principal objetivo o reparo da espinha bífida para preservação do tecido local e a reconstituição anatômica, minimizando assim riscos de infecção. ${ }^{1}$

Posteriormente são realizadas outras intervenções, se necessário, como a cirurgia para implantação da válvula de DVP, correção de bexiga e intestino neurogênico e ao longo do seu crescimento cirurgias para correção de deformidade músculos esqueléticos, tendo como finalidade a diminuição das sequelas decorrentes da mielomeningocele. ${ }^{8}$

A seguir os relatos dos voluntários:

\begin{abstract}
"30 cirurgias. A maioria para correção na perna, má formação. 2 correção da mielo na coluna, uma quando nasci e outra com 12 anos. Duas DVP, ampliação vesical" (Voluntario 1).
\end{abstract}

"Operação escoliose e lordose, nervos dos pés, válvula de derivação, e da Mielomeningocele" (Voluntario 2).

"Cerca de 25 entre correções ortopédicas nos pés, tornozelos, joelhos e quadris, cirurgias reparadoras e estéticas a além das cirurgias para contenção urinaria" (Voluntario 3).

"Ao todo foram 18 cirurgia, DVP, ampliação de bexiga por conta de pedras no rim, correção dos pés e joelhos" (Voluntario 4).

"Quatro procedimentos, correção da mielo, DVP, pés tortos e quadril" (Voluntaria 5).

Tendo em vista que algumas foram mais eficientes que outras, vimos como unanimidade à melhora da qualidade de vida após essas intervenções. Entretanto, a 
mobilidade dos membros inferiores foi à área mais afetada, tendo como maioria os procedimentos realizados ao longo do desenvolvimento para correção da marcha ou melhora mínima da mobilidade física. ${ }^{11}$

Além disso, os cinco voluntários realizaram correção cirúrgica das deformidades musculo esquelética, sendo possível observar na Tabela 3 a atual mobilidade motora dos voluntários.

Tabela 3 - Mobilidade Física motora.

\begin{tabular}{cccc}
\hline CATEGORIA & VARIÁVEL & NÚMERO $^{\circ}$ & $\%$ \\
\hline Mobilidade dos & Deambula & 1 & $20 \%$ \\
membros inferiores & $\begin{array}{c}\text { Deambula com auxílio (muletas, } \\
\text { bengala) }\end{array}$ & 2 & $40 \%$ \\
& Não deambula & 2 & $40 \%$ \\
& TOTAL: & 5 & $100 \%$ \\
\hline
\end{tabular}

Fonte: Dados subdivididos em categorias, a partir dos relatos coletados nas entrevistas, 2020.

Segundo Brandão (2009) "as deformidades variam conforme o segmento neurológico afetado; a retração no quadril e joelho e as deformidades nos pés ocorrem universalmente". Pois, quanto maior o nível ou o local onde está localizada a lesão, maior o comprometimento funcional e diminuição da força muscular dos membros inferiores. ${ }^{11,13}$

\section{Categoria 3. Apoio familiar no desenvolvimento}

A presença e apoio familiar são considerados de suma importância no desenvolvimento infantil, já que esta fase é caracterizada pelo aprimoramento e desenvolvimento de habilidades, desenvolvimento da base do caráter e principalmente da autoconfiança. ${ }^{14}$

Sendo assim, no desenvolvimento de uma criança com deficiência não seria diferente. Além disso, a decorrência destas condições crônica dos indivíduos com mielomeningocele abrangem diversas proporções, sejam elas emocionais, sociais e financeiras. Gerando impactos nas atividades rotineiras, tanto dele próprio como dos familiares envolvidos no seu cotidiano. ${ }^{15}$

Visando que tratamento e reabilitação desses indivíduos envolvem acompanhamento e cuidados contínuos devido às múltiplas intervenções que os mesmos são submetidos, o auxílio da família se torna fundamental no desenvolvimento do autocuidado e futuramente autonomia. ${ }^{14}$

Diante disso, vemos à importância do acompanhamento multidisciplinar, não só ao indivíduo com mielomeningocele, mas dos familiares envolvidos no seu cuidado, que em alguns casos por conta deste agravo acabam tornando-se super protetores, 
estimulando a dependência do (a) filho (a) deficiente aos familiares, podendo prejudicar o desenvolvimento do autocuidado, independência e integração social. ${ }^{16}$

A seguir o relato dos voluntários acerca do apoio familiar:

\begin{abstract}
"De forma totalmente normal, me apoiam e estão comigo em todas as decisões. Acabei mudando um pouco a família por ser a única pessoa com deficiência" (Voluntário 3).

"No começo eles ficaram bastante abalados, mas hoje em dia não. Viram que tinha tratamento pra amenizar os problemas e lidaram da melhor forma possível" (Voluntário 4).

"Eles sempre me ajudaram muito, me apoiam demais. Quando vim pro Brasil, eles ficaram meio receosos, falei que precisava buscar minha independência, eles aceitaram" (Voluntária 5).
\end{abstract}

\title{
Categoria 4. Principais obstáculos
}

Os principais obstáculos encontrados além do fator físico e as doenças secundárias são as psicológicas. Tendo em vista o preconceito referente a pessoas com deficiência, relacionado a mudanças nos padrões pré-estabelecidos pela nossa sociedade. ${ }^{14}$

Abaixo podemos identificar nitidamente os impactos que cada um sofre diariamente devido a sua deficiência:

\footnotetext{
"Na adolescência comecei a ter alguns problemas, com relação a preconceito. É normal, mas não deveria ser. Consegui lidar bem com isso" (Voluntário 1).
"Hoje em dia ainda existe muito pré-conceito com relação à inclusão de nós portadores de deficiência (PCD') acho que para mim melhorou muito em relação a isso mesmo existindo algum tipo de receio de outras pessoas a portar-se a nós" (Voluntario 2).

"Me considero apto a sociedade, passei e passo alguns preconceitos, mas fazem parte do dia a dia e precisam ser superados" (Voluntário 3).
}

De modo geral, quando retratamos o cotidiano dos indivíduos com alguma deficiência, é importante visualizar as limitações que os acompanham diariamente como consequência da mesma, acarretando na inserção social do mesmo para com a sociedade, fazendo com que deixem de realizar atividades pela falta de acessibilidade.

Entretanto, a evolução tecnológica ao longo dos anos resultou em avanços significativos das tecnologias assistivas, assim melhorando a qualidade de vida e 
inserção dos mesmos na sociedade, possibilitando adaptações para realização de atividades cotidianas e proporcionando acima de tudo independência. ${ }^{17}$

\begin{abstract}
"Na vida adulta, estou me adaptando, quando ia fazer alguma coisa e via que tinha dificuldades, buscava me adaptar com a minha condição física, então nunca me limitou" (Voluntário 1).

"Sim e não, busquei sempre não ver como limitador ter a mielo e sim como algo a mais para superar. Consegui realizar muitas das etapas sociais comuns da vida e as que não consegui foram por incapacidade de outras atribuições" (Voluntário 3).

"Limitou algumas vezes, por exemplo, quando queria fazer alguma coisa sozinha e não conseguia. Mas sempre procurei ser independente e ter minha própria autonomia "(Voluntária 5).
\end{abstract}

No entanto, poucos conhecem os direitos a acessibilidade e igualdade que está prevista em lei desde 2015. Na Lei n 13.146, de 06 de julho de 2015. Art.4 "Considerase discriminação em razão da deficiência toda forma de distinção, restrição ou exclusão, por ação ou omissão [...] incluindo a recusa de adaptações razoáveis e de fornecimento de tecnologias assistivas". Assim como, os direitos a saúde, educação, moradia, habilitação e reabilitação dos portares de qualquer deficiência estão em vigor e previstos em lei. ${ }^{18}$

\title{
Categoria 5. Desenvolvimento Profissional
}

Segundo o Art. 27, da Lei $n^{\circ} 13.146$, de 06 de julho de 2015 "a educação constitui direito da pessoa com deficiência, assegurando um sistema educacional inclusivo em todos os níveis e aprendizado ao longo de toda a vida". ${ }^{18}$

No entanto, literaturas revelam que durante o processo de escolarização ocorrem diferentes entraves, que vão desde o processo de matricula, acessibilidade à inserção social, mesmo com os direitos estando previstos em lei. ${ }^{19}$

A principal característica encontrada nos relatos dos voluntários abaixo foi que durante todo o desenvolvimento escolar e principalmente na primeira infância eles sofreram algum tipo de preconceito, através do bullyns por parte dos colegas ou pelo despreparo dos profissionais de educação quanto ao manejo de sua deficiência.

\footnotetext{
"Sofri preconceito, apenas quando eu era mais novo, o chamado bullyns no colégio, pelo fato de andar diferente dos demais" (Voluntário 4).

"Quando eu era criança tive uma barreira, de preconceito, porque queriam me mandar para um colégio especial sem necessidade. Eles não entendiam que meu problema era físico, não mental" (Voluntária 5).
} 
Entretanto, a partir dos relatos dos voluntários abaixo vemos que a realidade foi diferente no período acadêmico, os mesmos relataram boa aceitação por parte dos seus professores e colegas de classe, o fácil acesso e acessibilidade nas Universidades. Assim como, no mercado de trabalho após o término dos seus estudos, conseguindo seguir carreira na profissão almejada, mesmo depois de enfrentar os obstáculos impostos durante a educação na primeira infância.

\begin{abstract}
"Ensino médio regular. Iniciei duas vezes a faculdade e tranquei. Agora estou fazendo graduação a distância de Educação Física. Sou paratleta desde 2012, campeão nacional de para jiu-jítsu" (Voluntário 1).

"Trabalho e fiz faculdade de Ciências Contábeis, pretendo fazer mais alguma graduação" (Voluntário 2).

"Conclui meus estudos com louvor e passei a atuar antes mesmo da conclusão do curso de designer gráfico. Atualmente trabalho por conta e presto serviços voluntários na associação dos deficientes físicos do paraná, virei paratleta de esgrima em cadeira de rodas e nos ultimas dez anos conquistei dois campeonatos nacionais e cheguei a terceiro das Américas" (Voluntário 3).
\end{abstract}

"No mestrado, eles procuram sempre ajudar, estão reformado a sala para minha inclusão, sempre preocupados, por exemplo, de como vou andar pela sala com a cadeira" (Voluntária 5).

\title{
Categoria 6. Relacionamentos Afetivos
}

Ao discutir sobre os relacionamentos afetivos não falamos apenas de questões de sexualidade, namoro e casamento, mas as relações de amizade e familiares também. Essas relações são indispensáveis para o desenvolvimento da autoconfiança e interação social deste indivíduo com a sociedade. ${ }^{14}$

A sociedade de forma geral baseia a imagem corporal a partir de padrões já preestabelecidos, desencadeando frustrações quando algo foge deste padrão. Esse processo ocorre principalmente no período da adolescência, sendo portador de alguma deficiência ou não, pois é nesta fase que o desenvolvimento e entendimento acerca da autoestima ficam mais evidentes. Na fase da adolescência os indivíduos que portadores de necessidades especiais acabam encontrando ainda mais obstáculos, em relação à aceitação da própria imagem corporal e aceitação da própria condição. ${ }^{14}$

Quando retratado sobre relacionamentos afetivos, muitos relatam o preconceito que sofrem pelos seus parceiros por conta da sua deficiência, como retratado nos relatos abaixo. Este fator fica ainda mais evidente com as mulheres onde vemos maior influência de padrões de beleza, enquanto com os homens ficam relacionados à sua imagem corporal e desempenho. ${ }^{20}$

"Sim, existe preconceito, principalmente na geração na qual eu me encaixo. Preconceito em relação à aparência é bem nítido, evidente que existe preconceito. Mas hoje eu não me importo tanto. Este é um assunto bem delicado "(Voluntário 1). 


\begin{abstract}
"Sim, tenho 34 anos e não tive um relacionamento afetivo e devo grande parte desse fracasso a falta de confiança em meu corpo decorrente da mielo. A insegurança já é algo natural em um homem e atrelada a fatores que diminuem suas condições de efetiva ação física ou sexual aumentam em muito a chances de fracasso" (Voluntário 3).

"Sim, já tive situações complicadas. O preconceito por parte dos homens, eles desistem quando descobrem a sua deficiência. Meu ex-namorado era deficiente também, mas não aceitava, tivemos um problema em relação a minha deficiência e ele terminou. Atualmente namoro, ele é deficiente também e cadeirante, a gente se entende muito bem, sem problemas“ (Voluntária 5).
\end{abstract}

A partir dos relatos, podemos evidenciar o nítido desconforto dos voluntários ao retratar o assunto. Pois, apesar de muitos se aceitarem e conseguirem lidar com diversos obstáculos, notamos os preconceitos que os mesmos sofrem ou sofreram por conta da sua deficiência e o quanto isso impactou na autoestima e confiança.

Além disso, não há um suporte de assistência direto para lidar com esse fator psicológico, seja por falta de procura, disponibilidade ou até mesmo pela indisponibilidade de informações acerca do desenvolvimento adulto dos portadores de mielomeningocele.

\title{
CONSIDERAÇÕES FINAIS
}

Através dos dados coletados durante as entrevistas e interpretados a partir da literatura foi possível compreender as principais dificuldades ultrapassadas por adultos com mielomeningocele e como esta deficiência o impactou durante o seu desenvolvimento, principalmente em virtude das múltiplas intervenções cirúrgicas ou terapêuticas.

Percebeu-se a necessidade de medidas de inclusão, a partir dos relatos de preconceito enfrentado diariamente por conta da sua deficiência, principalmente durante o período escolar na primeira infância, tendo que lidar com os bullyng e com despreparo profissional dos educadores, tornando-se necessária uma maior orientação aos mesmos.

Além disso, é importante ressaltar a insatisfação diante da procura por publicações acerca de adultos portadores de mielomeningocele, sendo mais acessível à literatura sobre recém-natos e crianças com a doença. Entretanto, ao longo dos anos contamos com os avanços das tecnologias na assistência e a melhor desenvoltura dos profissionais da saúde acerca do tratamento, consequentemente o aumento da expectativa e qualidade de vida dos portadores desta deficiência, que a partir dos relatos coletados conseguimos constatar.

Tendo em vista as sequelas ocasionadas por essa malformação, conseguimos através dos relatos constatar uma melhora unânime após intervenções que os mesmos foram submetidos ao longo do desenvolvimento, proporcionando melhora na 
qualidade de vida e possibilitando a integração social. Porém, sem sucesso ao procurar literaturas recentes ou registros sobre esses avanços cirúrgicos e terapêuticos, que possibilitaram a melhor recuperação dos mesmos.

\section{REFERÊNCIAS}

1.Bizzi JWJ, Machado A. Mielomeningocele: conceitos básicos e avanços recentes. J Bras Neurocirurg, Porto Alegre, 2012; 23 (2): 138-151.

2. Baldisserotto CM, Kondo LHT, Chamlian, TR. Perfil epidemiológico dos pacientes com Mielomeningocele do centro de reabilitação Lar Escola São Francisco. Med. Reabil, São Paulo, 2010; 29(3); 76-9.

3. Rocco FM, Saito ET, Fernandes AC. Acompanhamento da locomoção de pacientes com mielomeningocele da Associação de Assistência à Criança Deficiente (AACD). ACTA FISIATR, São Paulo, 2007; 14(03): 126-129.

4. Affonso CV, Sonatti JG. Nutrição e o Ciclo da Vida, Gravidez, Amamentação e a Criança Pré-Escolar. 2014.

5. Linhares AO, Cesar JA. Suplementação com ácido fólico entre gestantes no extremo Sul do Brasil: prevalência e fatores associados. Ciência \& Saúde Coletiva, Rio Grande do Sul, 2017; 22(2):535-542.

6. Castilho SD, Filho AAB. Crescimento pós-menarca. Arquivos Brasileiros de Endocrinologia \& Metabologia 2000 jun.; 44(3).

7. Castro SC, Moraes DCG, Martins THP, Comácio SM. Estudo retrospectivo de casos de Disrafismo Espinhal no Hospital de Clínicas da Universidade Federal de Uberlândia (HCUFU). Biosci. J, Uberlândia, 2010 July/Aug; 26(4): 653-660.

8. Salomão JF, Pinheiro JAB, Carvalho JGS, Leibinger RD, Lucchesi G, Bomfim V. Mielomeningocele: tratamento cirúrgico e resultados. Jornal de Pediatria, 1995; 71(6).

9. Oliveira MF, Pinto FCG. Derivação ventriculosinusal retrógrada em lactentes com hidrocefalia após correção de mielomingocele. Catálago USP. São Paulo, 2016.

10. Yeh WSC, Kiohara PR, Soares ISC, Carmona MJC, Rocha FT, Galvão CES. Prevalência de Sinais de Sensibilidade ao Látex em Pacientes com Mielomeningocele Submetidos a Múltiplos Procedimentos Cirúrgicos. Revista Brasileira de Anestesiologia; 2012 jan./fev.; 62(1).

11. Ferreira FR, Bexiga FP, Martins VVM, Favero FM, Sartor CD, Artilheiro MC, Voos MC. Independência em crianças com mielomeningocele. Fisioter Pesqui. 2018; 25(2):196-201. 
12. Brandão AD, Fujisawa DS, Cardoso JR. Características de crianças com mielomeningocele. Fisioter. Mov. 2009 jan/mar; 22(1): 69-75.

13. Luz CL, Moura MCDS, Becker KK, Teixeira RAA, Voos MC, Hasue RH. The relationship between motor function, cognition, independence and quality of life in myelomeningocele patients. Arq Neuropsiquiatr; 2017.

14. Maia ACB. A Importância das relações familiares para sexualidade e autoestima de pessoas com Deficiência Física. O portal dos Psicólogos; 2010.

15. Gaiva MAM, Neves AQ, Siqueira FMG. O cuidado da criança com espinha bífida. Esc Anna Nery Rev. Enferm 2009 Out/Dez; 13 (4): 717-25.

16. Freitas GL, Senab RR, Silva JCF, Castro FFS. Reabilitação de crianças e adolescentes com mielomeningocele: o cotidiano de mães cuidadoras. Rev Gaúcha Enferm, 2016.

17. França ISX, Pagliuca LMF, Baptista RS. Política de inclusão do portador de deficiência: possibilidades e limites. Acta Paul Enferm. 2008; 21(1): 112-6.

18. Brasil. Lei $n^{\circ} 13.146$, de 6 de jul. de 2015. Institui a Lei Brasileira de Inclusão da Pessoa com Deficiência (Estatuto da Pessoa com Deficiência). Brasília, DF, jul 2015. Disponível em: <http://www.planalto.gov.br/ccivil_03/_ato20152018/2015/lei/112 146.htm>.

19. Assis CP, Martinez CMS. O perfil de necessidades especiais apresentados pelos alunos com sequelas de mielomeningocele incluídos no ensino regular. $\mathrm{V}$ Congresso Brasileiro Multiprofissional de Educação Especial. Londrina, 2009.

20 Soares AHR, Moreira MCN, Monteiro LMC. A qualidade de vida de jovens portadores de espinha bífida brasileiros e norte-americanos. Ciência \& Saúde Coletiva 2008; 13.

Artigo recebido em: $31 / 03 / 2020$

Artigo aprovado em: 03/05/2021

Artigo publicado em: 11/05/2021 